Please do not remove this page

RMIT

UNIVERSITY

\title{
A BDI game master agent for computer role-playing games
}

Luong, Bao; Thangarajah, John; Zambetta, Fabio; Hasan, Md Mahmud

https://researchrepository.rmit.edu.au/esploro/outputs/9921858791701341/filesAndLinks?institution=61RMIT_INST\&index=null

Luong, B., Thangarajah, J., Zambetta, F., \& Hasan, M. M. (2013). A BDI game master agent for computer role-playing games. Proceedings of the 12th International Conference on Autonomous Agents and Multiagent Systems (AAMAS 2013), 1187-1188.

https://researchrepository.rmit.edu.au/discovery/fulldisplay/alma9921858791701341/61RMIT_INST:Resea rchRepository

Document Version: Published Version

Repository homepage: https://researchrepository.rmit.edu.au

(c) 2013, International Foundation for Autonomous Agents and Multiagent Systems

Downloaded On 2023/04/26 19:55:59 +1000 
Thank you for downloading this document from the RMIT Research Repository.

The RMIT Research Repository is an open access database showcasing the research outputs of RMIT University researchers.

RMIT Research Repository: http://researchbank.rmit.edu.au/

\section{Citation:}

Luong, B, Thangarajah, J, Zambetta, F and Hasan, M 2013, 'A BDI game master agent for computer role-playing games', in Takayuki Ito, Catholijn Jonker, Maria Gini, Onn Shehory (ed.) Proceedings of the 12th International Conference on Autonomous Agents and Multiagent Systems (AAMAS 2013), Minnesota, United States, 6-10 May 2013, pp. 1187-1188.

See this record in the RMIT Research Repository at:

https://researchbank.rmit.edu.au/view/rmit:23882

Version: Published Version

\section{Copyright Statement:}

(C) 2013, International Foundation for Autonomous Agents and Multiagent Systems

Link to Published Version:

http://www.aamas-conference.org/Proceedings/aamas2013/docs/p1187.pdf 


\title{
A BDI Game Master Agent for Computer Role-Playing Games.
}

\section{(Extended Abstract)}

\author{
Bao Vo Luong, John Thangarajah, Fabio Zambetta and Mahmud Hasan \\ School of Computer Science and IT, RMIT University, Melbourne, Australia \\ \{bao.luong,john.thangarajah,fabio.zambetta,mahmud.hasan\}@rmit.edu.au
}

\begin{abstract}
In this paper we describe an approach for developing an intelligent game master (GM) for computer role-playing games. The role of the GM is to set up the game environment, manage the narrative flow and enforce the game rules whilst keeping the players engaged. Our approach is to use the popular Belief-Desire-Intention (BDI) model of agents to developing a GM.
\end{abstract}

\section{Categories and Subject Descriptors \\ D.2.10 [Software Engineering]: Design-methodologies}

\section{Keywords}

BDI Agent Systems, Computer Role-Playing Games, Game Mastering.

\section{INTRODUCTION AND MOTIVATION}

In recent years, computer games and interactive storytelling research have been emphasising the need for a rational 'coordination agent' that orchestrates the behaviour of several autonomous characters according to some highlevel, often plot-oriented, policy without taking direct control of all their actions. Examples of such a trend have been narrative controllers or director agents in interactive storytelling [2], AI directors in computer games [1] and, directly inspired from real-life, game masters in a table-top or liveaction Role-Playing Game (RPG) [3].

A Game Master (GM) in table-top RPGs is a person in charge of organising engaging game sessions for cooperative multi-player experiences, usually in the range of 4-8 participants. The GM describes the events taking place in the game's fictional world, she gives life to the characters populating it, and then finally communicates the outcomes of players' decisions, enforcing any game rules as needed.

A table-top RPG game session is often regarded as an emergent theatrical production where players take the role of lead actors and the Game Master serves as the director that provides stage, scenery and a flexible plot that adapts as a result of the interaction between players and the fictional game world. Examples of interaction between Game Masters and players abound in specialised press, game books,

Appears in: Proceedings of the 12th International Conference on Autonomous Agents and Multiagent Systems (AA$M A S$ 2013), Ito, Jonker, Gini, and Shehory (eds.), May, 6-10, 2013, Saint Paul, Minnesota, USA.

Copyright (C) 2013, International Foundation for Autonomous Agents and Multiagent Systems (www.ifaamas.org). All rights reserved. academic publications as well as dedicated web sites ${ }^{1}$ and on-line gaming communities.

Our work contributes a solution to the design and implementation of an automated GM agent for computer roleplaying games. The GM agent will set up the game environment, manage the narrative flow in a flexible fashion and it will enforce the game rules as needed while keeping the players engaged. Such a GM agent will help address issues in current computer role-playing games, which resort to scripting every possible event in the game's narrative. Not only does this lead to a substantial increase in design and programming time for the game [5], but it also reduces the narrative flexibility, which will have a negative impact on players' engagement.

Our design uses the Belief-Desire-Intention (BDI) model of agents to flexibly pursue the goals of the plot. BDI agents are designed and implemented using mental attitudes such as beliefs, goals, plans and intentions [4]. The use of such mental attitudes in software design allows developers to break complex decisions into smaller, more intuitive components. This technology is most useful when building systems that operate in highly dynamic environments as it allows for real-time online reasoning. The design of such systems allow incorporating alternatives strategies for achieving the one task, thus enabling flexible and robust systems. These traits, make BDI agent technology ideal for developing an automated software GM.

\section{DESIGNING A BDI GM AGENT}

There are 3 main steps in designing a BDI based GM agent: (i) Constructing a 'game-flow' diagram out of a sample game scenario; (ii) Constructing a 'goal tree' based on the game-flow diagram; and (iii) Constructing the agent's detailed 'goal-plan tree' by adding plans, to achieve the identified goals, and additional sub-goals as appropriate.

(i) Constructing the game-flow diagram: The first step, is to construct a game-flow diagram for the scenario. We begin with an abstract diagram as shown in Figure 1, where in order to Get inside the Hideout the player has to go through two main stages: Gain Access To Hideout Door and Player Opens Hideout Door. The game-flow diagrams for each of them were subsequently developed and they are shown, respectively, in Figure 2 and Figure 3.

(ii) Constructing the goal tree: In BDI agent design methodologies an initial task in the requirement analysis phase is to

\footnotetext{
${ }^{1}$ For instance, see http://www.darkshire.net/ ${ }^{j h k i m / ~}$ rpg/whatis/tabletop.html.
} 


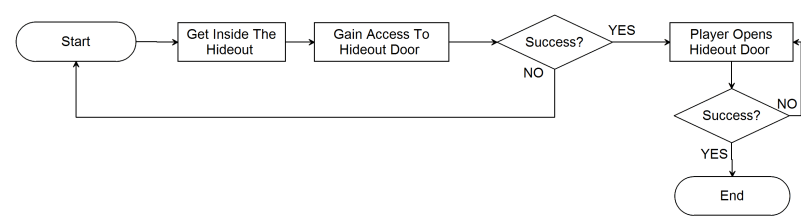

Figure 1: Game-flow diagram of the overall scenario.

identify the goals of the system. Each goal is decomposed into sub-goals which are further refined, leading to a goal tree structure. The second step to constructing our BDI GM agent is to develop this goal tree structure ${ }^{2}$.

We do this by transferring all of the operation-steps in the game-flow diagrams, developed as described above, to goals. All operation steps connected by a decision node in the game-flow diagrams become 'OR' connected sub-goals in the goal tree, 'AND' connected subgoals otherwise.

(iii) Constructing the detailed goal-plan tree: In general when designing a BDI agent system, after having identified the goals of the system, the goals are assigned to roles which are in turn assigned to agents. In this work, we are only concerned with a single agent; the game master agent. Hence, we avoid the need for detailing roles, and also inter-agent components such as communication protocols.

All the goals of the system are therefore assigned to the single GM agent. The next step is then to develop the internal details of the agent in terms of plan and subgoal structures to achieve the assigned goals.

We develop the detailed goal-plan tree using a two-phase approach: (i) first, propagate all the goals from the goal tree developed in the previous stage to an initial goal-plan tree with appropriate plan structures, ensuring coverage of all operation-steps from the game-flow diagrams; (ii) refine the goal-plan tree to enhance run-time flexibility and robustness.

\section{EVALUATION}

We implemented a prototype GM for a particular scenario of the Neverwinter Nights computer RPG. We used this prototype to evaluate the effectiveness of our BDI-based GM agent. The human participants that played both, the game with the BDI GM and without, found that the BDI GM provided greater replayability, flexibility, and clearer game objectives. They also found the version with the BDI GM more interesting overall and was the unanimous choice for future play. The construction of the game-flow diagrams was informed by observations made while playing the scenario multiple times, as well as by excerpts of the game progression contained in the Neverwinter Nights Official Adventure Guide.

\section{CONCLUSION}

As future work, we intend to investigate how to engineer knowledge about different scenarios in such a way that the GM can be more easily pluggable across many, without the need for heavy modifications. Clearly, knowledge elicitation is a crucial issue for the GM which we have not discussed in this paper. We envisage that while some of the knowledge could be generalised and will thus be domain-independent,

\footnotetext{
${ }^{2}$ In the Prometheus methodology this corresponds to the goal-overview diagram.
}

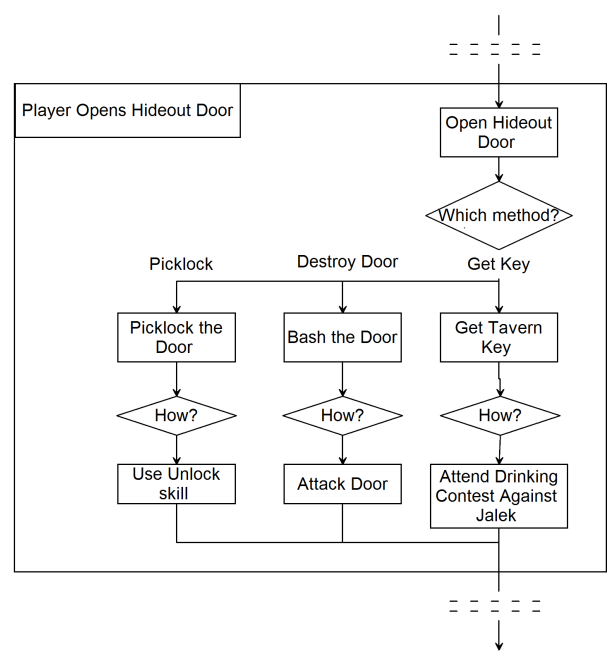

Figure 2: Details of the Gain Access To Hideout Door phase in the original scenario.

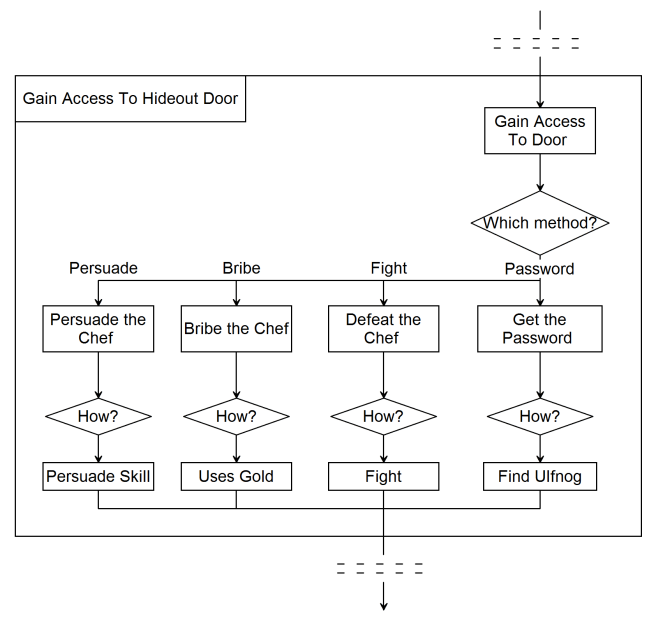

Figure 3: Details of the Player Opens Hideout Door phase in the original scenario.

there will certainly be some dependency on the domain (scenario). We would also consider incorporating knowledge about the player into the GM, possibly learning player preferences.

\section{REFERENCES}

[1] M. Booth. The AI systems of Left 4 Dead. Invited talk at the AIIDE 2009 conference.

[2] M. Mateas and A. Stern. Façade: An experiment in building a fully-realized interactive drama. Invited talk at the Game Developers Conference 2003.

[3] F. Peinado and P. Gervas. Transferring game mastering laws to interactive digital storytelling. In Technologies for Interactive Digital Storytelling and Entertainment, Lecture Notes in Computer Science, pages 48-54. Springer, 2004.

[4] A. S. Rao and M. P. Georgeff. BDI agents: From theory to practice. In In proceedings of the first international conference on multi-agent systems (ICMAS-95), pages 312-319, 1995.

[5] F. Zambetta. Implementing story-driven games with the aid of dynamical policy models. In S. Rabin, editor, AI Game Programming Wisdom 4, pages 551-570. 2008. 